\title{
Análise Espectral para suporte à Implantação de Rede de Campus baseada em Rádio Cognitivo sobre Faixas de TV UHF
}

\author{
Gabriel W. A. Silva ${ }^{1}$, Andson M. Balieiro ${ }^{2}$, Kelvin L. Dias ${ }^{1}$ \\ ${ }^{1}$ Centro de Informática- Universidade Federal de Pernambuco (UFPE) \\ 50670-901-Recife - PE - Brasil \\ ${ }^{2}$ Universidade de Pernambuco (UPE) - Campus Garanhuns \\ \{gwas, kld\}@cin.ufpe.br, andson.balieiro@upe.br
}

\begin{abstract}
This paper presents an analysis on UHF TV spectrum occupation as a support for deployment of an indoor and outdoor Cognitive Radio-based campus network at Federal University of Pernambuco, Brazil. Different from previous works, spectrum measurements campaigns and analysis on propagation models for building of geo-location databases were performed considering indoor and outdoor environments. The adopted methodology may be applied to different scenarios. Results show the feasibility of deploying an opportunistic campus network in UHF TV bands (75\% of availability) and that the selection propagation model may vary according to the location and environment type, where three approaches were presented.
\end{abstract}

Resumo. Este trabalho apresenta uma análise sobre a ocupação da faixa de TV UHF na cidade do Recife como suporte a implantação de uma rede de campus indoor e outdoor baseada em Rádio Cognitivo na Universidade Federal de Pernambuco. Diferente de outros trabalhos, campanhas de medição de espectro e análise sobre modelos de propagação para a construção da Base de Dados Geolocalizados de espectro foram conduzidas considerando ambientes indoor e outdoor. A metodologia apresentada é aplicável a outros cenários. Resultados mostram a viabilidade de implantação da rede de campus oportunista na faixa de TV UHF (75\% de disponibilidade) e que a definição do modelo de propagação pode variar de acordo com o local e tipo de ambiente, onde três abordagens foram apresentadas.

\section{Introdução}

A demanda por comunicação móvel é crescente e a expectativa é que, em 2020, seja 200 vezes maior que a atual [Jiang and Liu 2017]. Para atender a esta demanda, a disponibilidade de espectro eletromagnético e o seu uso eficiente são essenciais. Entretanto, tem-se verificado que grandes porções do espectro estão subutilizadas ou não utilizadas, principalmente, aquelas destinadas aos sistemas de TV [Barnes et. al

This work was supported by the research cooperation project between Motorola Mobility (a Lenovo Company) and CIn-UFPE. 
2016]. Um dos fatores que contribuem para isso é a atual política de alocação espectral estática [Mauwa et. al 2016], que concede ao chamado usuário primário - UP (ex. operadoras de telefonia, TV e rádio) o uso exclusivo de uma faixa de frequência por um intervalo de tempo (ex. anos). Assim, a revisão desta política torna-se imperativa [Barnes et. al 2016] e uma solução consiste na adoção da política de acesso dinâmico ao espectro (DSA), onde a tecnologia de rádio cognitivo $(\mathrm{RC})$ é vista como uma habilitadora para sua implantação [Balieiro et. al 2014].

Com DSA, ao usuário denotado como usuário secundário (US) é permitido acessar oportunistamente o espectro licenciado para realizar a sua comunicação, ou seja, enquanto o UP não o estiver utilizando. Desta forma, o US deve ser capaz de identificar se uma dada faixa espectral está disponível (o UP está ausente) antes de utilizá-la, bem como, liberá-la caso o UP reapareça, de modo a não interferir na comunicação primária. Para isso, duas principais abordagens têm sido consideradas: o sensoriamento de espectro e o uso de base de dados geolocalizados de espectro (GLDBs) [Balieiro et. al 2014]. Na primeira, o US realiza uma varredura no espectro para determinar os canais disponíveis. Isto demanda recursos de hardware não presentes nos dispositivos móveis atuais e pode sofrer com fenômenos como o problema do terminal escondido [Mauwa et.al 2016]. Na segunda, o US consulta o GLDB para obter a lista de canais disponíveis. O GLDB utiliza informações como localização do US, tipo de dispositivo, transmissores primários e modelos de propagação para definir esta lista. Um fator importante para o bom desempenho desta abordagem é a seleção adequada do modelo de propagação.

Cada vez mais as empresas e universidades estão oferecendo conexão sem fio aos seus usuários através de redes de campus. Em geral, a implantação de tais redes tem adotado a tecnologia Wi-Fi devido ao baixo custo dos dispositivos e operação em banda não licenciada desta tecnologia. Entretanto, quando situada na freqüência de $2,4 \mathrm{GHz}, o$ Wi-Fi pode ter seu desempenho degradado devido à interferência gerada por sinais de outras tecnologias tais com o Bluetooth e Zigbee, que atuam nesta mesma faixa. Operando em $5 \mathrm{GHz}$, menor interferência externa é sentida, porém as características de propagação deixam o sinal mais sensíveis aos obstáculos e demandam um número maior de pontos de acesso (APs) para a implantação da rede [Mauwa et. al 2015].

Com a mudança do sinal de TV analógico para o digital, novas oportunidades de transmissões podem ser alcançadas nas faixas espectrais VHF (Very High Frequency) e UHF (Ultra High Frequency), também conhecidas como TVWS (TV White Spaces)[Barnes et. al 2016]. Isso pode ser uma alternativa para a implantação de redes de campus e melhoria do seu serviço através do uso oportunista de tais faixas, já que elas apresentam melhores características de propagação do que as do Wi-Fi. No Brasil, este processo de mudança iniciou em março de 2016, na cidade de Rio Verde-GO, e ocorreu em Recife-PE, no mês de julho de 2017 [Anatel 2017].

A análise espectral das faixas de TV é um importante passo para a implantação de uma rede de campus em TVWS. Ela busca identificar o nível de ocupação das faixas espectrais e as oportunidades para transmissão oportunista e provê subsídios para a decisão de qual o modelo de propagação mais adequado para a construção do GLDB [Barnes et. al 2016]. Além disso, como em uma rede de campus (ex. de uma universidade) existe demanda por conexão móvel tanto em ambiente indoor (e.g. laboratórios, departamentos, salas de aula) quanto outdoor, esta análise deve ser 
realizada considerando estes dois tipos de ambientes. Neste aspecto, este trabalho apresenta uma análise sobre a ocupação espectral na faixa de TV UHF na cidade do Recife como suporte à implantação de uma rede de campus baseada em rádio cognitivo na Universidade Federal de Pernambuco (UFPE) para ambientes indoor e outdoor. Campanhas de sensoriamento de espectro e análise sobre modelos de propagação para a construção do GLDB da rede foram conduzidas. Diferente de outros trabalhos [Islam et. al 2008; Ying et. al 2013; Mauwa et. al 2015; Mauwa et.al 2016; Barnes et. al 2016], nós realizamos medição e análise de ocupação espectral e dos modelos de propagação para ambos os ambientes (indoor e outdoor). A metodologia apresentada neste trabalho é aplicável a outros cenários. Além disso, os resultados mostram a viabilidade de implantação da rede de campus de forma oportunista na faixa de TV UHF $(75 \%$ de disponibilidade) e que a definição do modelo de propagação mais suscetível de utilização no GLDB pode variar de acordo com o local e tipo de ambiente, onde três abordagens foram elencadas, destacando suas vantagens e desvantagens.

Diante do que foi delineado, este trabalho encontra-se assim organizado. A Seção 2 apresenta os trabalhos relacionados. Seção 3 descreve a metodologia adotada nas campanhas de medição. Os resultados obtidos são apresentados na Seção 4. A Seção 5 conclui este artigo.

\section{Trabalhos Relacionados}

Diversos trabalhos têm analisado a ocupação espectral em diferentes regiões do mundo, setores do espectro e ambientes [Islam et. al 2008; Ying et. al 2013; Mauwa et. al 2015; Mauwa et.al 2016; Barnes et. al 2016]. Por exemplo, em [Islam et. al 2008], os autores realizam medições no espectro em Singapura (em pontos outdoors) para identificar os padrões de uso das frequências entre $80 \mathrm{MHz}$ e $5,85 \mathrm{GHz}$ e bandas subutilizadas. Com o mesmo objetivo, em [Barnes et. al 2016], uma campanha de medição foi realizada nas bandas de TV (VHF e UHF) no campus da universidade de Pretória, África do Sul, em seis pontos (4 outdoors e 2 indoors). Nele, a análise de oportunidades de espectro indoor e outdoor para um mesmo ponto não é possível, pois em cada ponto apenas um tipo de medição é tomado. Embora as medições tenham sido feitas na universidade, o trabalho não visou à implantação de redes de campus. Já em [Ying et. al 2013], os autores realizam medição do espectro tanto em ambiente indoor quanto outdoor na cidade de Hong Kong para entender as diferenças entre os padrões de uso das faixas espectrais nestes dois tipos de ambientes. Em geral, eles observam uma grande porcentagem de faixas subutilizadas, que no ambiente indoor é maior que no outdoor. Desta forma, eles propõem um sistema de identificação de faixas subutilizadas para ambiente indoor. Diferente de nosso trabalho, ambos [Barnes et. al 2016; Ying et. al 2013] não realizam análise de modelos de propagação para a construção de GLDB. Além disso, aqueles trabalhos focam em medições em área metropolitana.

Em [Mauwa et.al 2016], uma análise sistemática sobre o sensoriamento de espectro e uso de GLDBs é conduzida. Os autores argumentam que não existem modelos de propagação claramente definidos para regiões em desenvolvimento. Deste modo, eles analisam diversos modelos de propagação em comparação com resultados de medição de espectro coletados na cidade do Cabo, na África do Sul. Embora alguns aspectos da metodologia sejam similares ao empregado no nosso trabalho, três pontos claramente os distinguem: os autores somente avaliam ambientes outdoors; restringem a 
análise aos modelos de propagação, sem tratar da porcentagem de faixas espectrais disponíveis; o cenário de medição é de área metropolitana. Sob a motivação de rede de campus, em [Mauwa et.al 2015], medições de espectro nas faixas de TV na área do campus da universidade do Cabo, na África do Sul, são realizadas. Os autores adotam dois pontos de medição externos e buscam quantificar as disponibilidades de faixas existentes para uso oportunista. Embora a medição seja em área de campus, nenhuma análise sobre ocupação espectral em ambientes internos ou modelos de propagação para a construção de GLDBs é realizada.

\section{Metodologia}

Como indicado na Seção 1, o uso das faixas TV (TVWS) pode ser uma alternativa para implantação/melhoria de redes de campus através do uso de RC. Por exemplo, considerando o padrão IEEE 802.11af, taxas de transmissão de até $12 \mathrm{Mbps}$ podem ser alcançadas, e as melhores características de propagação das faixas de TV aproveitadas. Mas, para que isso seja possível, dois pontos são importantes: analisar o nível de ocupação da faixa espectral de TV a fim de verificar o grau oportunidades para emprego do DAS e implantação da rede de campus; definir o mecanismo para identificação dessas oportunidades pelo usuário secundário, evitando causar interferência no UP.

No segundo ponto, o uso de GLDBs tem sido sugerido para identificação das oportunidades e considerado como um requisito para utilização de TVWS pela Comissão Federal de Comunicações (FCC) dos EUA [Ying 2013]. Em geral, tais GLDBs estimam o estado do espectro baseado em modelos de propagação [Mauwa et.al 2016]. Assim, o desempenho alcançado pelo sistema oportunista é fortemente impactado pelo modelo de propagação utilizado no GLDB e a decisão de qual modelo utilizar é uma questão-chave na implantação desse sistema. Além disso, como as redes de campus oferecem comunicação móvel em ambientes indoor e outdoor, tanto a análise do nível de ocupação quanto à do modelo de propagação a ser adotado no GLDB devem considerar estes dois tipos de ambientes.

Nesta seção apresentamos a metodologia adotada nas análises para prover suporte à implantação de redes de campus baseadas em RC na UFPE para ambientes indoor e outdoor utilizando faixas de TV UHF. Assim, 4 pontos de medição distintos na UFPE são considerados, onde para cada um, medições indoor e outdoor são realizadas. Deste modo, as duas análises seguintes são conduzidas.

- Análise da ocupação espectral de canais de TV UHF: avalia o nível ocupação de 38 canais de TV na faixa $470 \mathrm{MHz}-698 \mathrm{MHz}$ em Recife, indicando o grau de disponibilidade de utilização oportunista de tais canais pelos usuários secundários oriundos de uma rede de campus.

- Análise de modelos de propagação: examina quatro modelos de propagação (perdas de percurso) para construção de um GLDB. Para isso, medições do sinal de TV Digital (UHF) pertencente a um canal de TV aberta local e informações do transmissor foram utilizadas na análise. Esta análise busca denotar qual modelo fornece resultados mais próximos dos valores medidos e, assim, mais suscetível de ser adotado na construção de GLDBs para a rede de campus da UFPE. 


\subsection{Equipamentos Adotados no Sensoriamento}

No sensoriamento da faixa de TV UHF foram adotadas uma placa USRP (Universal Software Radio Peripheral) N210 e uma antena LP0410 [Ettus 2017]. Esta antena permite transmissão e recepção de sinais na faixa de $400 \mathrm{MHz}$ a $1000 \mathrm{MHz}$. Neste trabalho, apenas a função de recepção foi utilizada. O método de sensoriamento por detecção de energia foi implementado usando GNU Radio [GNU Radio 2017] em um notebook Intel Core i5-3230M CPU $2.60 \mathrm{GHz}$ com sistema operacional Ubuntu conectado a USRP. A Figura 1 ilustra os dispositivos adotados nas medições. Além deles, um nobreak foi usado nas medições externas (ambiente outdoor).

O sensoriamento por detecção de energia foi configurado para atuar na faixa de 400 a $1000 \mathrm{MHz}$, com resolução (granularidade) de $6,250 \mathrm{~Hz}$ e tamanho da transformada rápida de Fourier (FFT) igual a 160. Deste modo, 96000 medições de potência de sinal foram coletadas para cada varredura total da faixa, com tempo médio de varredura de 6,5 minutos. Todos os canais de TV apresentam $6 \mathrm{MHz}$ de largura de banda

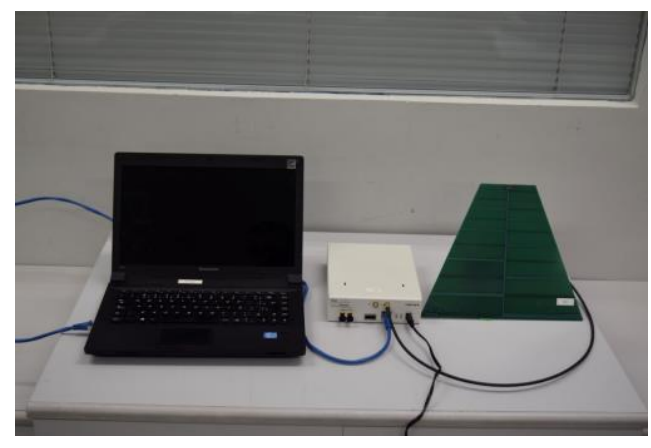

Figura 1. Equipamentos usados para o sensoriamento espectral

\subsection{Informações do Transmissor de TV}

Para analisar os modelos de propagação quanto à viabilidade de adoção dos mesmos na construção da base de dados de TVWS (GLDB), considerando ambientes indoor e outdoor, um estudo de caso com um canal de TV foi definido. Assim, medições do sinal de TV Digital (UHF) pertencente a um canal de TV aberta local (Rede Globo Nordeste) foram coletadas para serem comparadas aos valores provenientes dos modelos de propagação.

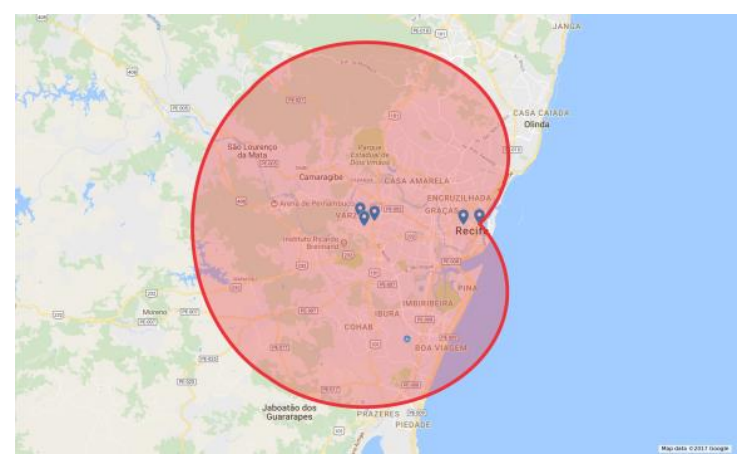

Figura 2. Esquema de dissipação do sinal de TV 
O transmissor rádio base do sinal está localizado no Bairro de Santo Amaro, em Recife-PE (Latitude $8^{\circ} 3^{\prime} 16,1^{\prime \prime}$ S e Longitude $34^{\circ} 52^{\prime} 41,3$ 'W). A torre de transmissão possui 137 metros de altura, com antenas de irradiação próximas ao topo. A transmissão do sinal ocorre na frequência central de $605,143 \mathrm{MHz}$ (canal 36 da faixa de TV UHF), com potência de $30 \mathrm{~kW}$. Informação de altitude do terreno também foi utilizada para efeito de cálculos dos modelos de propagação. A antena da emissora em questão segue um modelo de irradiação cardióide (ver Figura 2) direcionando a potência máxima do sinal para uma direção de ângulo aberto. Neste aspecto, todos os pontos de medição foram escolhidos no sentido da maior dissipação da potência do sinal da antena, os quais são apresentados na Seção 4.3.

\subsection{Localização dos Pontos de Medição}

As campanhas de sensoriamento foram conduzidas na Universidade Federal de Pernambuco (UFPE), Recife, Brasil. Três pontos foram escolhidos com a finalidade de formar um triângulo envolvendo o Campus e, assim, captar as peculiaridades da irradiação dos sinais em pontos extremos da UFPE. Eles são apresentados a seguir.

- Reitoria - UFPE: sala localizada no térreo de um prédio de três andares e distante $7,34 \mathrm{~km}$ da fonte do sinal. Situa-se na latitude $8^{\circ} 3^{\prime} 8^{\prime \prime} \mathrm{S}$ e na longitude $34^{\circ} 56^{\prime} 41^{\prime \prime} \mathrm{W}$ e tem 14 metros de altitude. É o ponto mais próximo da antena do transmissor. As medições indoors ocorreram na altitude de $15 \mathrm{~m}$ e as externas foram feitas no estacionamento do prédio, distante em torno de $15 \mathrm{~m}$ do ponto de medição indoor.

- Centro de Informática (CIn-UFPE): sala localizada no segundo andar do prédio

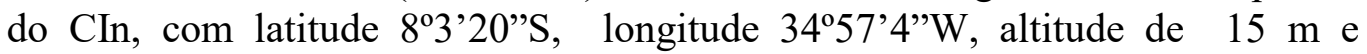
distante $8,05 \mathrm{~km}$ da fonte de sinal. As medições indoors foram tomadas na altitude de $23 \mathrm{~m}$. Já as medições outdoors ocorreram no estacionamento do mesmo prédio, distante quase $10 \mathrm{~m}$ do ponto de medição interna.

- Centro de Filosofia e Ciências Humanas (CFCH - UFPE): sala do segundo andar do prédio do $\mathrm{CFCH}$, distante $8,35 \mathrm{Km}$ do transmissor. Possui latitude de $8^{\circ} 3$ ' 1 's, longitude de $34^{\circ} 57^{\prime} 14^{\prime \prime} \mathrm{W}$ e altitude de 16 metros. É o ponto mais distante em relação ao transmissor da Globo Nordeste. As medições indoors foram tomadas a $25 \mathrm{~m}$ de altitude e as externas no estacionamento do $\mathrm{CFCH}$, distante em torno de $8 \mathrm{~m}$ do ponto de medição interna.

Além desses, para a análise dos modelos de perdas de percurso (propagação), a definição de um ponto de referência $d_{0}$ foi necessária. Este ponto foi o seguinte:

- Universidade Católica de Pernambuco (Unicap): estacionamento distante 1,22 $\mathrm{km}$ do transmissor, com latitude $8^{\circ} 3^{\prime} 17^{\prime \prime} \mathrm{S}$, longitude $34^{\circ} 53^{\prime} 18^{\prime \prime} \mathrm{W}$ e 10 metros de altitude. É o ponto mais próximo da fonte de sinal.

Neste último ponto, apenas medições outdoor foram realizadas para não afetar os cálculos que utilizam os dados deste ponto de medição. A Figura 3 ilustra a posição geográfica dos pontos de medição e a Tabela 1 sumariza as localizações. 
Tabela 1. Localização dos pontos de medição

\begin{tabular}{|c|c|c|c|}
\hline Nome do Local & $\begin{array}{c}\text { Latitude/ } \\
\text { Longitude }\end{array}$ & Altitude (m) & $\begin{array}{c}\text { Distância do transmissor } \\
(\mathrm{Km})\end{array}$ \\
\hline Unicap (Ponto do) & $8^{\circ} 3^{\prime} 17^{\prime \prime} \mathrm{S} / 34^{\circ} 53^{\prime} 18^{\prime \prime} \mathrm{W}$ & 10 & 1,22 \\
\hline Reitoria (UFPE) & $8^{\circ} 3^{\prime} 8^{\prime \prime} \mathrm{S} / 34^{\circ} 56^{\prime} 41^{\prime \prime} \mathrm{W}$ & 14 & 7,34 \\
\hline CIn (UFPE) & $8^{\circ} 3^{\prime} 20^{\prime \prime} \mathrm{S} / 34^{\circ} 57^{\prime} 4^{\prime \prime} \mathrm{W}$ & 15 & 8,05 \\
\hline CFCH (UFPE) & $8^{\circ} 3^{\prime} 1^{\prime \prime} \mathrm{S} / 34^{\circ} 57^{\prime} 14^{\prime \prime} \mathrm{W}$ & 16 & 8,35 \\
\hline
\end{tabular}

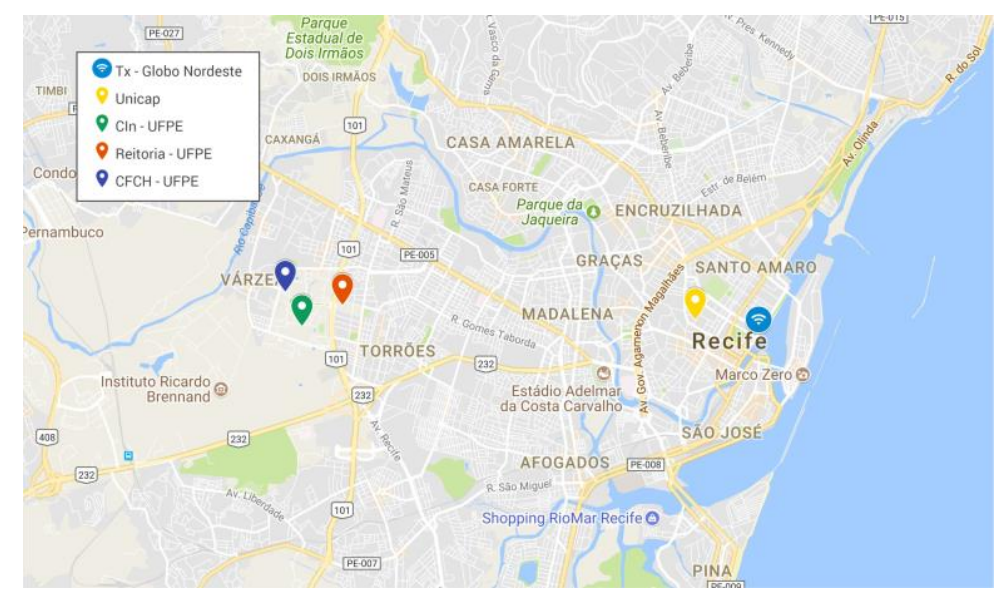

Figura 3. Localização dos pontos de medição

Para verificar que a distância entre o ponto $d_{0}$ e a antena da Globo Nordeste é adequada, ou seja, não é pequena para que o efeitos da difração de campo próximo aconteçam, duas regras foram conferidas: $d \gg \lambda$ e $d \gg D$, onde $d$ é a distância da região far-field, isto é, a distância mínima para a qual as regras de irradiação são válidas, $D$ é a maior distância linear das dimensões da antena e $\lambda$ é o comprimento de onda do sinal emitido pela fonte. Sendo o comprimento de onda a divisão entre a velocidade da luz no vácuo $\left(\sim 300 \times 10^{6} \mathrm{~m} / \mathrm{s}\right)$ e a frequência do sinal $(605,143 \mathrm{MHz})$, tem-se que $\lambda$ é aproximadamente $0,5 \mathrm{~m}$. Considerando que a antena de transmissão ocupe $10 \%$ do comprimento (altura) da torre que a suporta, ou seja, $D>13,7 \mathrm{~m}$, conclui-se que $d \gg 0,5 m$ e $d \gg 13,7 m$. Dado que um valor muito maior ( $\gg$ ) seja pelo menos dez vezes o valor referencial, tem-se que $d \geq 140 \mathrm{~m}$. Além disso, para definir a distância $d$ é necessário considerar o diagrama de irradiação da antena, de forma que a potência do lóbulo central de propagação do sinal seja capturada. A Figura 4 ilustra um exemplo do diagrama de irradiação do sinal de uma antena de TV.

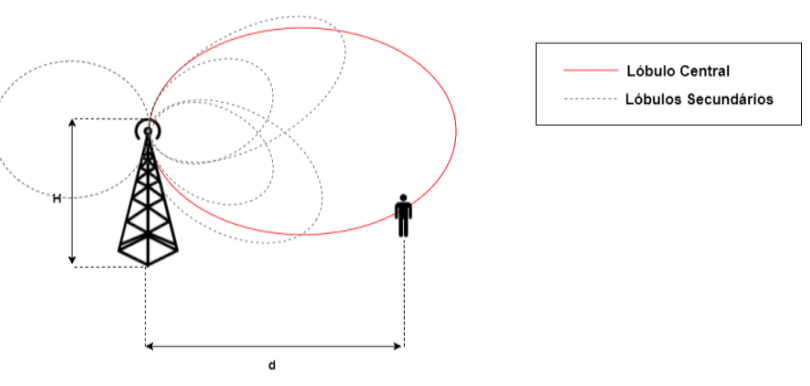

Figura 4. Exemplo de diagrama de irradiação de sinal de uma antena de TV 
Embora as antenas de TV Digital tenham lóbulo central com ângulos mais abertos, é necessário definir uma margem de segurança para que os dados medidos sejam condizentes com a situação real. Deste modo, considerando que há um ângulo maior que $70^{\circ}$ entre o sinal irradiado e a torre da antena (em relação ao usuário), então este necessita estar distante da torre no mínimo duas vezes a altura dela $(\mathrm{H})$ para conseguir captar os sinais do lóbulo central, ou seja, $d>2 x H=2 x 137=274 \mathrm{~m}$. Desta forma, a distância mínima para o ponto $d_{0}$ é em torno de $280 \mathrm{~m}$. Como o ponto $d_{0}$ escolhido dista $1,22 \mathrm{Km}$ do transmissor de TV, então esta condição também é satisfeita.

\subsection{Modelos de Propagação}

Em comunicação sem fio nem sempre é possível obter o estado das transmissões no espectro em tempo real. Para contornar isso, modelos de propagação têm sido propostos na literatura, onde informações sobre perda de percurso do sinal e potência do sinal recebido podem ser estimadas, por exemplo. Em geral, os modelos de propagação apresentam baixo custo computacional [Sakar et. al 2003] e auxiliam os sistemas na estimação do estado atual do espectro. Eles têm sido empregados na construção dos GLDBs para determinar a disponibilidade de espectro [Ying et. al 2013]. Neste trabalho, quatro modelos de propagação foram analisados, os quais são:

- Modelo de Perda no Espaço Livre (FSPL): é descrito como a perda do nível de sinal de uma onda eletromagnética que se assemelha a um caminho de linha de visão através do espaço livre, sem obstáculos próximos para causar reflexão ou difração do sinal [Rappaport 2001]. A Eq. 1 define a perda de percurso do sinal (em $\mathrm{dB})$, onde $d$ é a distância entre o transmissor e o receptor $(\mathrm{em} \mathrm{km}), f$ é a frequência do sinal (em GHz). A base logarítmica das equações ao longo do texto é a decimal, exceto quando indicado diferente.

$$
F S P L=20 \log d+20 \log f+92,45
$$

- Hata: é empregado para predição de sinais em áreas urbanas. É um modelo empírico cuja perda de percurso média é dada pela Eq. 2 [Sakar et. al 2003], onde $f$ é a frequência do sinal (em MHz), que varia entre $150 \mathrm{MHz}$ e 1500 $\mathrm{MHz}, h_{t}$ e $h_{r}$ são as alturas da antena do transmissor e do receptor (em metros), $d$ é a distância entre o transmissor e o receptor (em $\mathrm{km}$ ) e o valor de $a$ é o fator de correção da altura da antena do receptor, que é dado pela Eq. 3 para pequenas e médias cidades.

$$
\begin{gathered}
P L_{\text {Hata }}=69,55+26,16 \log f-13,82 \log h_{t}-a\left(h_{r}\right)+\left(44,9-\log h_{t}\right) \log d \\
a\left(h_{r}\right)=(1,1 \log f-0,7) h_{r}-(1,56 \log f-0,8) d B
\end{gathered}
$$

- Ericsson 9999: é uma extensão do modelo Hata e ajusta os parâmetros segundo o ambiente de propagação. A perda de percurso para áreas urbanas é dada pelas Eqs. 4 e 5 [Milanovic et. al 2010], com $f$ em MHz e $h_{t}, h_{r}$ e $d$ em metros.

$$
P L_{\text {Ericsson }}=36,2+30,2 \log d-12 \log h_{t}+0,1 \log h_{t} \log d-3,2\left[\log \left(11,75 h_{r}\right)\right]^{2}+g(f)
$$




$$
g(f)=44,49 \log f-4,78(\log f)^{2}
$$

- Egli: É um modelo de terreno para propagação de radio frequência [Chebil et. al 2011] aplicável a sistemas que atuam na faixa de 40-900 MHz, cuja perda de percurso é obtida pela Eq. 6, com $h_{t}$ e $h_{r}$ em metros, $d$ em km e $f$ em MHz.

$$
P L_{E g l i}=20 \log f+40 \log d-20 \log h_{t}+76,3-10 \log h_{r}
$$

\subsection{Definição do Limiar de Detecção}

A definição do limiar (threshold) de detecção é um dos parâmetros importantes no sensoriamento de espectro. Este limite determina a partir de qual nível de potência percebida, o canal é considerado ocupado. Isso impacta na aferição de disponibilidade do canal e nas comunicações primária e secundária. Caso um limiar muito baixo seja escolhido, o sistema secundário pode se tornar excessivamente conservador (proteção excessiva ao UP) e gerar alarmes falsos frequentes, indicando que o UP está presente, quando na verdade o canal está vago. Isto ocasiona perdas de oportunidades de utilização oportunista do espectro pelo US. Por outro lado, o uso de limiar elevado pode ocasionar perda de detecção do UP e interferência excessiva no UP, pois o US considera o canal como disponível e o utiliza, quando na verdade o canal está ocupado pelo UP.

Não há um consenso na literatura sobre o melhor limiar a adotar. Segundo a FCC, o valor ideal de limiar para sinais de TV Digital é de $-114 \mathrm{dBm}$ (canais de $6 \mathrm{MHz}$ ) [8]. Porém, este limite é considerado conservador por [Ying et. al 2013], que define um limiar de $-85,5 \mathrm{dBm}$ para canais de TV Digital de $8 \mathrm{MHz}$. Buscando um compromisso entre proteção ao UP e identificação de oportunidades de uso do espectro, utilizou-se um limiar intermediário igual a $-107 \mathrm{dBm}$, similar ao adotado em [Mauwa et. al. 2015].

\section{Resultados}

Esta Seção apresenta os resultados obtidos para as duas análises indicadas na Seção 3. Para cada ponto de medição e tipo de ambiente, 1,25 horas de sensoriamento na faixa de $(470 \mathrm{MHz}-698 \mathrm{MHz})$ foram conduzidas. Cada varredura na faixa completa durou aproximadamente 3 minutos.

\subsection{Premissas dos Modelos de Propagação}

Antes do inicio da análise dos dados medição, a validade da lei da perda quadrática de potência com a distância para os pontos de medição da rede de campus foi verificada. Para isso, a equação de transmissão de Friis [Rao 2007] foi usada (ver Eq. 7), que calcula o nível de potência teórico no receptor distante $d$ metros do transmissor, onde $\mathrm{P}_{\mathrm{r}}\left(d_{0}\right)$ é a potência recebida no ponto de referência, distante $d_{0}$ metros da antena.

$$
\mathrm{P}_{\mathrm{r}}(d)=\mathrm{P}_{\mathrm{r}}\left(d_{0}\right)+20 \log \left(d / d_{0}\right)
$$

Com a potência recebida no ponto mais próximo (referência) da antena $\mathrm{P}_{\mathrm{r}}\left(d_{0}\right)$, foi possível calcular a potência recebida teórica nos outros pontos de medição, situados na UFPE. A Tabela 2 apresenta os níveis de potência recebida teórica $\left(P_{r, T e o}\right)$ e medida $\left(P_{r, \text { Med }}\right)$ considerando os ambientes outdoor e indoor. Nota-se que na Reitoria da UFPE houve a maior diferença entre os valores medidos e teóricos. Isso ocorreu, pois o local 
apresenta poucos prédios no entorno. Assim, os efeitos de desvanecimento multicaminho e sombreamento foram mais fracos nessa localidade, permitindo que a intensidade do sinal recebido fosse maior. Além disso, nota-se que em média a diferença entre a intensidade do sinal medida e a teórica é aproximadamente 9, similar ao encontrado em [Mauwa et. al 2016], que adota pontos de medições com distâncias similares as adotadas no nosso trabalho, mostrando que a lei em questão é adequada para o cenário avaliado.

Tabela 2. Comparação entre os níveis de potência recebida teórica e medida nos ambientes outdoor e indoor.

\begin{tabular}{|c|c|c|c|c|c|c|c|}
\hline & \multicolumn{3}{|c|}{ Outdoor } & \multicolumn{3}{c|}{ Indoor } \\
\hline Local & $\begin{array}{c}\mathrm{d} \\
(\mathrm{Km})\end{array}$ & $\begin{array}{c}P_{r, \text { Med }} \\
(\mathrm{dBm})\end{array}$ & $P_{r, \text { Teo }}$ & $P_{r, \text { Med }}-P_{r, \text { Teo }}$ & $\begin{array}{c}P_{r, \text { Med }} \\
(\mathrm{dBm})\end{array}$ & $\begin{array}{c}P_{r, \text { Teo }} \\
(\mathrm{dBm})\end{array}$ & $P_{r, \text { Med }}-P_{r, \text { Teo }}$ \\
\hline Unicap $(d o)$ & 1,22 & $-82,25$ & - & - & $-82,25$ & - & - \\
\hline Reitoria-UFPE & 7,34 & $-81,78$ & $-97,84$ & 16,06 & $-107,51$ & $-98,95$ & $-8,56$ \\
\hline CIn-UFPE & 8,05 & $-92,15$ & $-98,64$ & 6,49 & $-95,65$ & $-99,75$ & 4,10 \\
\hline CFCH-UFPE & 8,35 & $-87,88$ & $-98,96$ & 11,08 & $-91,58$ & $-100,07$ & 8,49 \\
\hline
\end{tabular}

Para determinar a perda de percurso real nos experimentos, parâmetros como a potência efetiva isotrópica irradiada (EIRP) do transmissor e ganhos das antenas necessitam ser conhecidos. Entretanto, eles não estavam disponíveis. Para contornar essa situação e permitir a análise dos resultados obtidos, considerou-se que a perda de percurso no ponto $d_{0}$ é igual à perda no espaço livre [Mauwa et. al 2016].

\subsection{Resultados dos Modelos de Propagação versus Medições de Espectro}

Com a consideração anterior e as medições do nível de potência recebida, a perda de percurso do sinal de TV nos diferentes pontos de medição da UFPE foi calculada através da Eq.8, onde $\mathrm{P}_{L}\left(d_{0}\right)$ é a perda de percurso no ponto $d_{0}, M_{0} \mathrm{e} M_{i}$ são as potências do sinal (do transmissor) medidas nos pontos $d_{0}$ e $i$, respectivamente. As Tabelas 3 e 4 apresentam os valores (médios) de perda de percurso obtidos através dos dados medidos e dos modelos de propagação para os ambientes outdoor e indoor, respectivamente.

$$
\mathrm{P}_{L}(i)=\left(M_{0}-M_{i}\right)+\mathrm{P}_{L}\left(d_{0}\right)
$$

Tabela 3. Perda de percurso teórica e medida para o ambiente outdoor

\begin{tabular}{|c|c|c|c|c|c|}
\hline Local (i) & Medição & FPSL & Ericsson 9999 & Egli & Hata \\
\hline Unicap (Ponto $d o$ ) & 89,81 & 89,81 & 87,99 & 83,83 & 108,43 \\
\hline Reitoria (UFPE) & 89,34 & 105,4 & 108,18 & 110,78 & 128,89 \\
\hline CIn (UFPE) & 99,71 & 106,2 & 108,11 & 110,58 & 128,84 \\
\hline CFCH (UFPE) & 95,44 & 106,52 & 108,21 & 110,69 & 128,94 \\
\hline
\end{tabular}

Tabela 4. Perda de percurso teórica e medida para o ambiente indoor

\begin{tabular}{|c|c|c|c|c|c|}
\hline Local (i) & Medição & FPSL & Ericsson 9999 & Egli & Hata \\
\hline Unicap (Ponto $d o$ ) & 89,81 & 89,81 & 87,99 & 83,83 & 108,43 \\
\hline Reitoria (UFPE) & 113,96 & 105,4 & 107,72 & 110,14 & 128,44 \\
\hline CIn (UFPE) & 102.1 & 106,2 & 105,74 & 107,43 & 126,47 \\
\hline CFCH (UFPE) & 98,03 & 106,52 & 105,65 & 107,34 & 126,39 \\
\hline
\end{tabular}


Para melhor comparar os resultados, o erro médio (absoluto) para cada par (medição(M) e modelo de propagação) foi calculado. As Tabelas 5 e 6 sumarizam os valores para ambos os ambientes. Nota-se que em todos os pontos de medição, o FSPL apresentou melhor resultado para o ambiente outdoor, enquanto que o Ericsson 9999 foi superior em ambientes indoors. Além disso, em geral, os modelos conseguiram melhores resultados nos ambientes internos do que externos. Isto ocorreu porque nas medições externas, o nível potência detectado foi por volta de $9 \%$ maior que nas medições internas e a perda de percurso obtida nos modelos foi maior do que a obtida nas medições externas.

Tabela 5. Erro absoluto médio e desvio padrão (em dB) em ambiente outdoor

\begin{tabular}{|c|c|c|c|c|}
\hline Local (i) & M e FSPL & M e Ericsson 9999 & M e Egli & M e Hata \\
\hline Unicap (Ponto $d o$ ) & 0 & 1,82 & 5,98 & 18,62 \\
\hline Reitoria (UFPE) & 16,06 & 18,84 & 21,44 & 39,55 \\
\hline CIn (UFPE) & 6,49 & 8,4 & 10,87 & 29,13 \\
\hline CFCH (UFPE) & 11,08 & 12,77 & 15,25 & 33,5 \\
\hline Média & $\mathbf{8 , 4 1}$ & $\mathbf{1 0 , 4 6}$ & $\mathbf{1 3 , 3 8}$ & $\mathbf{3 0 , 2}$ \\
\hline
\end{tabular}

Tabela 6. Erro absoluto médio e desvio padrão (em dB) em ambiente indoor

\begin{tabular}{|c|c|c|c|c|}
\hline Local (i) & M e FSPL & M e Ericsson 9999 & M e Egli & M e Hata \\
\hline Reitoria (UFPE) & 8,56 & 6,24 & 3,82 & 14,48 \\
\hline CIn (UFPE) & 4,1 & 3,64 & 5,33 & 24,37 \\
\hline CFCH (UFPE) & 8,49 & 7,62 & 9,31 & 28,36 \\
\hline Média & $\mathbf{7 , 0}$ & $\mathbf{5 , 8 3}$ & $\mathbf{6 , 1 5}$ & $\mathbf{2 2 , 4 0}$ \\
\hline
\end{tabular}

Dos resultados (Tabelas 5 e 6), visualizamos diversas abordagens para construção do GLDB em termos da quantidade de modelos de propagação: (1) modelo único geral, (2) modelo único para cada tipo de ambiente, (3) modelo baseado no local de medição e tipo de ambiente. Na primeira, o FSPL seria o modelo mais apropriado para utilização, pois apresenta (em média) o menor valor do erro médio absoluto. Embora omitido deste artigo, o erro quadrático médio (MSE) obtido com este modelo também é menor. Considerando a abordagem 2, os modelos FSPL e Ericsson 9999 seriam os mais adequados para os ambientes outdoor e indoor, respectivamente. $\mathrm{Na}$ última abordagem, nota-se a predominância do FSPL para o ambiente externo, enquanto os modelos Ericsson 9999 e Egli seriam os adotados no ambiente interno, conforme sumarizado na Tabela 7.

Tabela 7. Melhor modelo de propagação para cada ambiente e local

\begin{tabular}{|c|c|c|}
\hline \multirow{2}{*}{ Local } & \multicolumn{2}{|c|}{ Ambiente } \\
\cline { 2 - 3 } & Outdoor & Indoor \\
\hline Reitoria-UFPE & FSPL & Egli \\
\hline CIn-UFPE & FSPL & Ericsson 9999 \\
\hline CFCH-UFPE & FSPL & Ericsson 9999 \\
\hline
\end{tabular}

A escolha da abordagem deve considerar o desempenho e o custo (complexidade) de cada uma. Por exemplo, optando pela primeira, a construção do GLDB é mais simples, pois apenas um modelo é empregado para estimar o estado do espectro em todos os pontos e tipos de ambiente. Entretanto, o desempenho pode ser deteriorado, pois para um dado ponto o mesmo nível de potência será estimado para ambos os ambientes, não considerando as suas peculiaridades. Caso, a abordagem 3 
fosse adotada, melhor fidelidade com o estado real do espectro poderia ser alcançada. Entretanto, ocasionaria maior complexidade ao processo de implantação do GLDB.

\subsection{Resultados da Ocupação Espectral}

Para analisar o nível ocupação espectral das faixas de TV, a métrica de ocupação relativa $\left(O R_{c}\right)$ foi adotada [Mauwa et. al. 2015] e é definida pelas equações em (9), onde $N_{k, c, t h}$ é a diferença entre a medição $k$ realizada no canal $c\left(S S_{k, c}\right)$ e o limiar de detecção th, $\overline{N_{c, t h}}$ e $M_{c, t h}$ são a média dos valores de $N_{k, c, t h}$ e o seu valor máximo, respectivamente, considerando $K$ medições.

$$
O R_{c}=\frac{100 \times \overline{N_{c, t h}}}{M_{c, t h}} ; \quad \overline{N_{c, t h}}=\frac{\sum_{k=1}^{K} N_{k, c, t h}}{K} ; \quad M_{c, t h}=\max _{k}\left(N_{k, c, t h}\right) ; \quad N_{k, c, t h}=S S_{k, c}-t h
$$

As Figuras 5 e 6 apresentam os resultados obtidos. Dos resultados, nota-se um número elevado de canais de TV ociosos, aproximadamente $75 \%$ deles com níveis de ocupação iguais ou próximos de zero. Isto indica um grande potencial de implantação de uma rede de campus baseada em rádio cognitivo, com uso oportunista dos canais. Notase ainda que na medição indoor da Reitoria-UFPE (Fig. 5-esquerda), para o canal 36, há uma ocupação relativa de aproximadamente $22 \%$, enquanto na outdoor esse valor alcança $100 \%$. Isso mostra que efeitos como sombreamento e terminal escondido podem ter afetado as medições internas neste ponto. Percebe-se fenômeno similar em outros 10 canais nesse ponto de medição. Essa diferença na indicação de disponibilidade pode ser tratada através da definição do limiar de detecção de acordo com o tipo de ambiente.
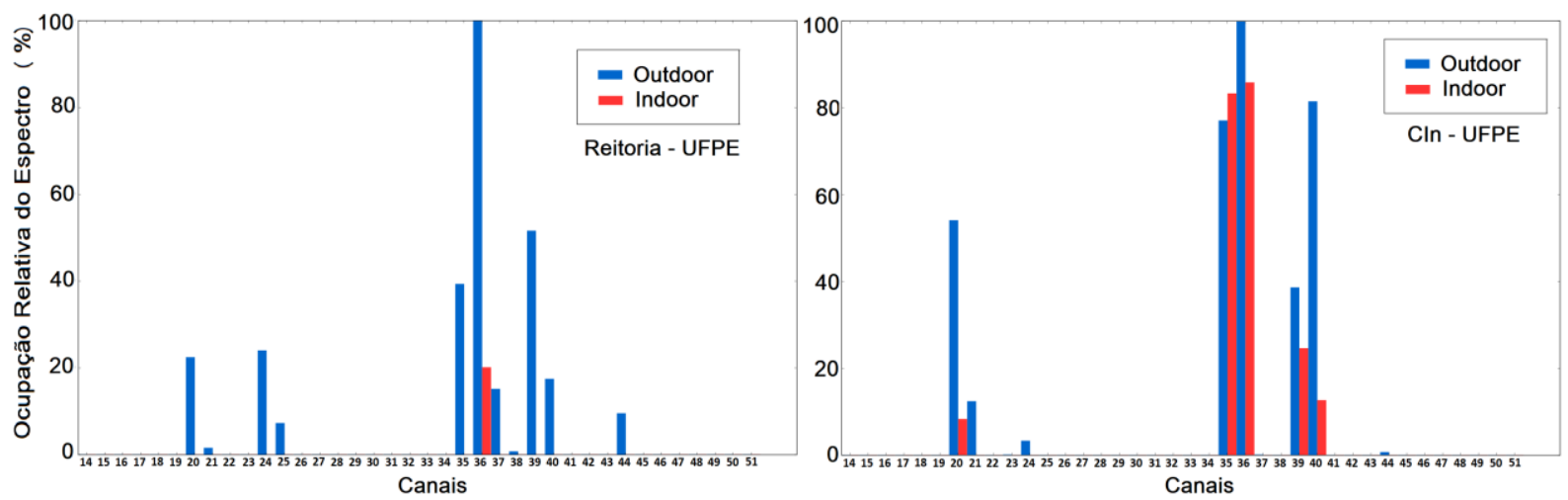

Figura 5. Ocupação Relativa do Espectro na Reitoria -UFPE (à esquerda) e ClnUFPE (à direita)

No CIn-UFPE (Fig. 5- direita) houve uma certa paridade nas medições indoor e outdoor nos canais 35 e 36, que indicaram os canais como ocupados na maior parte do tempo. Em geral, nota-se uma maior ocupação relativa nas medições externas do que nas internas. Isto é esperado, pois em áreas externas, o sinal recebido tem maior intensidade, devido a um menor número de barreiras presentes neste ambiente (paredes, prédios, por exemplo). Além disso, este ponto de medição apresentou maiores valores de ocupação relativa, indicando um nível maior atividade primária no entorno.

Condições como o nível do piso do prédio interno em que a medição ocorre, visão direta e ângulo de recepção do sinal podem ocasionar situações onde o nível de 
potência de um sinal no ambiente indoor é maior do que no outdoor. Esta situação pode ser observada no canal 35 da Fig. 6 (CFCH-UFPE), onde a ocupação relativa do canal no ambiente indoor é superior ao do outdoor. Isso pode ser resultado das medições internas terem sido realizadas no segundo piso do prédio do $\mathrm{CFCH}$, que apresenta uma melhor visada para o transmissor do que o estacionamento. Além disso, neste ponto de medição, nota-se um maior número de canais com ocupação relativa diferente de zero, o que indica maior detecção da atividade primária.

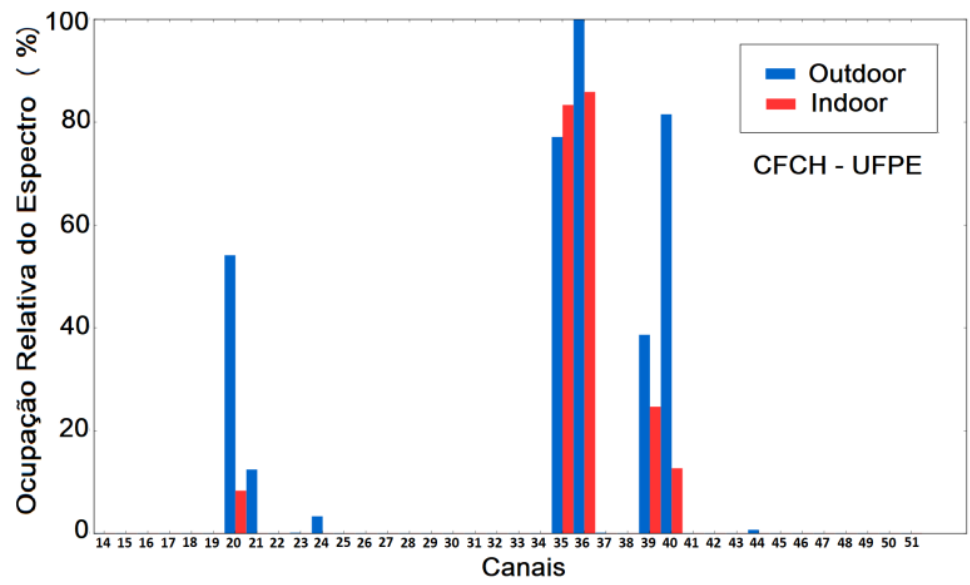

Figura 6. Ocupação Relativa do Espectro no CFCH -UFPE

\section{Conclusão}

Este artigo apresentou uma análise espectral como suporte à implantação de uma rede de campus baseada em RC para ambientes indoor e outdoor na UFPE. Para isso, campanhas de sensoriamento e análise sobre modelos de propagação para a construção do GLDB da rede foram conduzidas. Os resultados mostraram a existência de um número elevado de canais de TV subutilizados tanto em ambiente indoor quanto outdoor, os quais podem ser explorados para a implantação de uma rede de campus baseada em RC. Além disso, notou-se que a definição do modelo de propagação adotado na construção do GLDB é uma questão importante e que aspectos como tipo de ambiente e local (área) de atuação (onde a medição foi tomada) devem ser considerados na seleção. Neste aspecto, três abordagens foram elencadas, destacando as vantagens e desvantagens de cada uma. Como trabalho futuro pretende-se criar um protótipo da rede de campus com o uso de placas USRPs em um dos pontos analisados. Assim, a construção do GLDB e a implementação de outras funções de RC são vislumbradas para testes de algoritmos e avaliação de aplicações. Além disso, análises de modelos de propagação voltados para ambientes indoor, de diferentes limiares de detecção do UP e a combinação de informações do GLDB com informações locais de sensoriamento para aumentar a acurácia da detecção do UP são direções futuras.

\section{Referências}

Anatel (2017). "Cronograma do Desligamento da TV analogical é modificado", disponível em http://www.anatel.gov.br/institucional/ultimas-noticiass/1580cronograma-de-desligamento-da-tv-analogica-e-modificado. Acesso em Dez de 2017. 
Balieiro, A., Dias, K., Yoshioka, P. Cavalcanti, D., Cordeiro, C. (2014), "A multiobjective genetic optimization for spectrum sensing in cognitive radio", Expert System with Applications, vol. 41, issue 15, p.3640-3650.

Barnes, S.D., Botha, P.R. and Marahaj, B.T. (2016) "Spectral occupation of TV broadcast bands: Measurement and analysis", Measurement, vol. 93p.272-277.

Chebil, J., Lwas, A.K., Islam, Md. R., Zyoud, A-H. (2011). “Comparison of Empirical Propagation Path Loss Models for Mobile Communications in the Suburban Area of Kuala Lumpur", 4th International Conference on Mechatronics.

Ettus (2017). "USRP Networked Series". Disponível em https://www.ettus.com/product/category/USRP-Networked-Series. Acesso em Dez de 2018.

GNU Radio (2017). "GNU Radio is a Free and Open Source Toolkit for Software Radio”. Disponível em https://www.gnuradio.org/. Acesso em Dez de 2018.

Islam, M. H., Koh, C.L., Oh, S. W., Qing, X., Lai, Y. Y., Wang, C., Liang., Y-C., Toh, B. E., Chin, F., Tan, G. L., Toh, W. (2008) "Spectrum Survey in Singapore: Occupancy Measurements and Analyses", 3rd International Conference on Cognitive Radio Oriented Wireless Networks and Communications (CrownCom), p.1-7.

Jiang, D. and Liu, G. (2017) "An Overview of 5G Requirements", In: 5G Mobile Communications, Springer International Publishing, p. 3-26.

Mauwa, H., Bagula, A. Zennaro, M. (2015) "Exploring TV White Spaces for Use in Campus Networks", International Conference on e-Infrastructure and e-Services for Developing Countries, p. 14-25.

Mauwa, H., Bagula, A. Zennaro, M., Pietrosemoli, E., Lysko, A and Brown, T. X. (2016) "Systematic Analysis of Geo-location and Spectrum Sensing as Access Methods to TV White Space", ITU Kaleidoscope Academic Conference.

Milanovic, J., Rimac-Drlje, S., Majerski, I. (2010). "Radio Wave Propagation Mechanisms and Empirical Models for Fixed Wireless Access Systems", Technical Gazzete, vol. 17, p.43-52.

Rao, S. (2007). "Estimating the Zigbee Transmission-Range Ism Band-Designers of Short-Range Wireless Devices in the $900-\mathrm{MHz}$ and 2.4-GHz Band Need to Understand What and How Parameters Affect the Transmission Range, EDN, vol. $52, \mathrm{n}^{\mathrm{o}} 11$, p. $67-74$.

Rappaport, T.S. (2001). "Wireless Communications: Principles and Practice", 2nd Edition, Prentice Hall.

Sarkar, T.K., Ji, Z., Kim, K., Medouri, A., Salazar-Palma, M. (2003). “A Survey of Various Propagation Models for Mobile Communication", IEEE Antennas and Propagation Magazine, vol. 45, issue 3, p. 51-82.

Ying, X., Zhang, J., Yan, L., Chen, Y., Zhang, G., Chen, M., Chandra, R. (2013), "Exploring Indoor White Spaces in Metropolises", International Conference on Mobile Computing \& Networking, p. 255-266. 